Disponível em:

http://editora.unoesc.edu.br/index.php/race

RACE, Joaçaba, v. 16, n. 4, p. 121-146, jan./abr. 2017

\title{
DIMENSÕES DE BRAND EXPERIENCE NO CONTEXTO BRASILEIRO
}

Brand experience dimensions in the Brazilian context

Simone Gurgel de Brito

E-mail: simonegurgel@uern.br

Doutora em Administração pela Pontifícia Universidade Católica do Paraná; Mestre em Engenharia de Produção pela Universidade Federal de Santa Catarina; Professora na Universidade Estadual do Rio Grande do Norte.

Adriana Martins de Oliveira

E-mail: adrimartinso@gmail.com

Doutora em Administração pela Pontifícia Universidade Católica do Paraná;

Mestre em Administração pela Universidade Potiguar;

Professora na Universidade Estadual do Rio Grande do Norte e na Universidade Potiguar.

Liana Holanda Nepomuceno Nobre

E-mail: liananobre@ufersa.edu.br

Doutora em Administração pela Pontifícia Universidade Católica do Paraná; Mestre em Administração pela Universidade Estadual do Ceará; Professora na Universidade Federal Rural do Semiárido.

Álvaro Fabiano Pereira de Macêdo

E-mail: alvarofabiano@ufersa.edu.br

Doutor em Administração pela Pontifícia Universidade Católica do Paraná; Mestre em Ciências Contábeis pela Universidade de Brasília; Professor na Universidade Federal Rural do Semiárido. Endereço para contato: Rua Francisco Mota, 572, Alto de São Manoel, 59625-900, Mossoró, Rio Grande do Norte, Brasil.

Renato Zancan Marchetti

E-mail: renato.zancan@pucpr.br

Doutor em Ciências de Gestão pela Ecole Des Hautes Etudes Commerciales, HEC,

França; Mestre em Administração de Empresas pela Fundação Getúlio Vargas; Professor na Pontifícia Universidade Católica do Paraná.

Artigo recebido em 24 de maio de 2016. Aceito em 27 de setembro de 2016. 


\section{Resumo}

No presente artigo teve-se como objetivo testar a escala de brand experience proposta por Brakus, Schmitt e Zarantonello (2009) em um contexto brasileiro, assim como comparar os resultados aos achados de outros estudos semelhantes já publicados. Quanto à metodologia, foi realizado um estudo quantitativo e descritivo de corte transversal, com aplicação de Análise Fatorial Confirmatória e teste de Análise de Variância (Anova). A experiência de marca foi testada a partir de quatro dimensões: sensorial, afetiva, comportamental e intelectual. Foram analisadas 18 marcas experimentais por 254 respondentes. O estudo mostrou que as duas dimensões que apresentaram maior impacto na experiência de consumo foram a dimensão sensorial e a afetiva. Percebeu-se que as marcas que apresentaram menor grau de diferenciação são aquelas de pouco valor agregado e/ou consumo imediato. As marcas que são conhecidas por conferir um determinado grau de status ao consumidor (com exceção da Ray-Ban), como veículos, itens de vestuário e eletrônicos foram categorizadas como altamente diferenciadas. As marcas únicas foram aquelas com forte apelo sensorial e/ou afetivo, como O Boticário, Nestlé, Ray-Ban e Google.

Palavras-chave: Marketing experiencial. Marcas. Brand experience.

\section{Abstract}

The present study aimed to test the brand experience scale proposed by Brakus, Schmitt and Zarantonello (2009) in a Brazilian context, as to compare the results to other published similar studies. Regarding the methodology, a quantitative and descriptive cross-sectional study was developed with application of Confirmatory Factor Analysis and Analysis of Variance (Anova). The brand experience was tested from four dimensions: sensory, affective, behavioral, and intellectual. Eighteen experimental brands were analyzed by 254 respondents. The study showed that the two dimensions of greater impact on consumer's experience were sensory and affective. It was noticed that the brands that showed lower differentiation are those of little added value and/or immediate consumption. Brands that are known to confer a degree of consumer status (except for Ray-Ban), such as vehicles, clothing, and electronic items were categorized as highly differentiated. The unique brands were those with strong sensory and/or affective appeal as $O$ Boticário, Nestlé, Ray-Ban and Google.

Keywords: Experiential marketing. Brands. Brand experience.

\section{INTRODUÇÃO}

A diversidade em produtos e serviços existente no mercado, bem como a complexidade e a heterogeneidade das relações de consumo têm gerado uma crescente demanda por pesquisas na área de marketing. A teoria sobre gestão de marcas, 
com forte desenvolvimento a partir dos anos 1980, teve abordagens multidisciplinares considerando-se aspectos tangíveis e intangíveis da marca. Os estudos sobre marcas têm envolvido diferentes áreas, como: economia, gestão estratégica, comportamento organizacional, pesquisa do consumidor, psicologia e antropologia, entre outras (HEDING; KNUDTZEN; BJERRE, 2009). Entre as mais relevantes contribuições para essa área, Keller (2003) menciona os estudos sobre personalidade da marca (AAKER, 1997), teoria do relacionamento com a marca (FOURNIER, 1998) e conceito de comunidade de marca (MUNIZ; O’GUINN, 2001).

Do ponto de vista gerencial, ainda há divergência quanto ao conceito de marca e a forma mais adequada para se medir o seu valor. Essa condição tem motivado novos e relevantes estudos teóricos e empíricos sobre o tema, em diferentes setores econômicos. Kapferer (2008) considera que a análise da influência da marca, bem como os seus reflexos na consolidação e na lucratividade das organizações representam um tema de grande interesse para os gestores. Muniz e Marchetti (2012) destacam que os estudos relacionados à marca e suas diferentes dimensões oferecem uma orientação para estratégias de marketing das organizações e auxiliam a tomada de decisões.

Entre as diferentes abordagens da gestão de marcas, os aspectos de relacionamento do consumidor com a marca foram discutidos originalmente por Fournier (1998). A autora estendeu a metáfora do relacionamento interpessoal para analisar a relação entre o consumidor e a marca, considerando que as pessoas se relacionam com as marcas da mesma forma que se relacionam com outras pessoas. De acordo com Breivik e Thorbjørnsen (2008), a análise do relacionamento com marcas ajuda a conhecer melhor as necessidades dos consumidores, compreender alguns indicadores de lealdade do consumidor com a marca e auxilia no desenvolvimento de produtos e no aprimoramento das atividades de marketing das empresas.

Fournier (1998) afirma que os relacionamentos entre as pessoas e as marcas são válidos, conforme o nível das experiências vividas pelos consumidores. A experiência do consumidor com a marca foi um tema abordado originalmente por Holbrook e Hirschman (1982), buscando entender o lado emocional e irracional do comportamento do consumidor, pela análise da experiência advinda de um conjunto de interações entre a empresa e os seus consumidores. Essa visão “experiencial” caracteriza-se pelo fluxo de fantasias, sentimentos e diversão associados ao processo de consumo, que, por sua vez, apresentava uma variedade de significados simbólicos, respostas hedônicas e elementos estéticos.

Brakus, Schmitt e Zarantonello (2009) afirmam que entender como os consumidores experimentam marcas é crítico para desenvolver estratégias de marketing 
para bens e serviços e que as experiências ocorrem nos mais diversos ambientes, quando os consumidores buscam, compram, recebem ou consomem os produtos ou serviços. Os autores definem experiência de marca ou brand experience como uma resposta subjetiva interna do consumidor (sensação, sentimento e cognição), além de reações comportamentais, evocadas pelos estímulos relacionados a uma marca. Schmitt, Brakus e Zarantonello (2015) defendem que as experiências que ocorrem durante o uso e o consumo de produtos de marca são, indiscutivelmente, os estímulos mais fortes para a recompra e a recomendação boca a boca, bem como recomendações on-line.

A literatura mostra um crescente interesse por estudos envolvendo brand experience, considerando múltiplas perspectivas. Alguns trabalhos apresentam o foco direcionado a medidas do construto, contribuindo para o desenvolvimento de escalas que buscam medir a experiência do consumidor com marcas, como o trabalho de Brakus, Schmitt e Zarantonello (2009), enquanto outros estudam a influência de brand experience em relação a outros construtos de marca, como: atitude, lealdade, credibilidade, qualidade do relacionamento ou valor da marca (IGLESIAS; SINGH; BATISTA-FOGUET, 2011; LEE; KANG, 2012; SHAMIM; BUTT, 2013).

Apesar do crescente número de trabalhos teóricos sobre brand experience, ainda são consideradas escassas as ferramentas destinadas a apoiar os gerentes de marketing em suas ações estratégicas. Shamim e Butt (2013) afirmam que tanto pesquisadores quanto gestores estão em busca de evidências empíricas substanciais sobre contextos que gerem experiências capazes de penetrar profundamente na memória de longo prazo dos consumidores e, posteriormente, possam influenciar suas percepções e atitudes em relação à marca. O desenvolvimento e a validação de escalas robustas de brand experience podem auxiliar os gestores na elaboração dos estímulos certos para a promoção de experiências positivas e memoráveis do consumidor com a marca.

Para medir a experiência dos consumidores com marcas, Brakus, Schmitt e Zarantonello (2009) desenvolveram uma escala contendo 12 itens, divididos em quatro dimensões: sensorial, afetiva, comportamental e intelectual. No presente estudo tem-se como objetivo testar a escala de brand experience, proposta pelos autores em um contexto brasileiro, com a inclusão de marcas de maior apelo ao consumidor brasileiro, assim como comparar os resultados aos de outros estudos semelhantes já publicados. Ressalta-se que, embora a escala já tenha sido utilizada no contexto nacional por Semprebom (2011), uma das dimensões propostas por Brakus, Schmitt e Zarantonello (2009) - a dimensão comportamental - foi desmembrada nas dimensões afetiva e intelectual no modelo validado. Por perceber a relevância dos aspectos 
referentes a padrões de consumo e estilo de vida, optou-se por eleger um conjunto de marcas reconhecidamente experimentais no âmbito brasileiro a fim de testar as dimensões de experiência de marca originalmente propostas por Brakus, Schmitt e Zarantonello (2009).

Além desta introdução, o artigo apresenta uma revisão da literatura que abrange o tema gestão de marcas e descreve a escala utilizada neste trabalho para medir brand experience. Em seguida, são apresentados os métodos de coleta e tratamento estatístico dos dados e conclui-se com a descrição e a análise dos resultados obtidos.

\section{REFERENCIAL TEÓRICO-EMPÍRICO}

O marketing experiencial é um conceito que apresenta pouca tradição na literatura acadêmica, resultando, inclusive, em visões opostas em relação ao seu significado. Pontes (2012) retrata que uma das consequências dessas visões contraditórias é que o termo marketing experiencial é utilizado de maneira equivocada, mais frequentemente com a utilização do termo designando outras ações de marketing, como eventos ou ações promocionais. As discussões sobre o tema são levantadas por estudiosos que criticam os modelos tradicionais de decisão de compra, que assumem o consumo como comportamento lógico, funcional e racional, deixando de enfatizar questões sensoriais e emocionais como itens relevantes para a decisão. Nessa perspectiva, o presente trabalho se pauta nas visões de autores como Holbrook e Hirschman (1982), Pine e Gilmore (1998) e principalmente no trabalho de Brakus, Schmitt e Zarantonello (2009) para avançar na contribuição ao tema em debate.

\subsection{O CONCEITO DE EXPERIÊNCIA NA PERSPECTIVA DOS CONSUMIDORES E DO MARKETING}

As experiências de marca ocorrem quando indivíduos se expõem a situações de consumo por meio da realização de compras de mercadorias, da prestação de serviços ou da simples busca por eles (HOLBROOK, 2000). A experiência tanto pode ser direta, caracterizada pelo contato físico, quanto indireta, quando o contato é virtual. Gentile, Spiller e Noci (2007) destacam que as experiências têm papel fundamental na determinação da preferência dos consumidores, influenciando suas decisões de compra.

As experiências de consumo são compostas por fatores vinculados a diversos aspectos da interação entre o consumidor e o produto ou serviço. Assim, as experiên- 
cias de consumo são consideradas multidimensionais e se caracterizam pelos aspectos hedônicos, como sentimentos, fantasias e diversão (HOLBROOK; HIRSCHMAN, 1982). Para Gentile, Spiller e Noci (2007), as dimensões que compõem a experiência do consumidor são: sensorial, emocional, cognitiva, pragmática, estilo de vida e relacional. Os autores apresentam ainda quatro tipologias de experiências: entretenimento, educacional, de fuga e estéticas. Já Schmitt (2002) apresentou cinco fatores que compõem as experiências: sensoriais, afetivos, cognitivos, físicos, comportamentais e relacionais, que originaram os cinco Modelos Experienciais estratégicos: sensorial, emocional, cognitivo, comportamental e relacional, também utilizados por Ding e Tseng (2015).

\subsection{BRAND EXPERIENCE}

Partindo de diferentes contribuições teóricas ao longo dos anos, Gentile, Spiller e Noci (2007) definiram brand experience como “[...] a experiência do consumidor, originada de um conjunto de interações entre um consumidor e um produto, uma companhia, ou parte da organização, que provoque uma reação.” Para Brakus, Schmitt e Zarantonello (2009), os consumidores estão submetidos a vários estímulos relacionados às marcas, como cores, designs, formas, entre outros. Esses estímulos fazem parte da identidade da marca. Para os autores, portanto, brand experience corresponde às respostas internas e subjetivas dos consumidores, como sensações, sentimentos, bem como respostas comportamentais, aos estímulos que são promovidos pelas marcas. Tanto Gentile, Spiller e Noci (2007) quanto Brakus, Schmitt e Zarantonello (2009) destacam que a experiência é estritamente pessoal e implica o envolvimento do consumidor em diferentes níveis (racional, emocional, sensorial, físico e espiritual).

O entendimento de como ocorre a experiência dos consumidores com as marcas é considerado crítico no desenvolvimento de estratégias de marketing. De acordo com Schmitt (2002), a experiência tem sua origem em uma observação direta ou participação em acontecimentos virtuais, reais ou até mesmo imaginários; ou seja, a experiência pode se originar tanto de uma interação material quanto de uma interação abstrata. Para Brakus, Schmitt e Zarantonello (2009), as experiências podem ocorrer em qualquer lugar, mesmo que o consumidor não tenha nenhum envolvimento anterior com a marca, pois a experiência envolve busca, compra, recebimento ou consumo de produtos ou serviços pelos consumidores. Quanto às formas de classificação, as experiências podem variar em força e intensidade, além de oscilar entre experiências positivas e negativas. Em relação ao tempo, as experiências podem ser curtas ou du- 
radouras, ficando, dessa forma, na mente do consumidor por mais tempo (BRAKUS; SCHMITT; ZARANTONELLO, 2009).

Uma vez que a oferta de produtos e serviços foi se tornando algo comum, a promoção da experiência surgiu como principal diferencial das marcas, transformando-se, assim, em um componente da estratégia das empresas (PINE; GILMORE, 1998). Diferentemente do marketing tradicional, o marketing experiencial não tem foco nas características e benefícios dos produtos, mas, sim, nas experiências oferecidas aos consumidores nas situações de consumo. Para tanto, o marketing deve concentrar esforços nas experiências que a empresa fornece aos clientes, apelando aos sentidos emocionais dos clientes e influenciando sua decisão de escolha. Essa concepção corrobora a ideia de Prahalad e Ramaswamy (2004); para eles, a empresa deve criar um ambiente propício para a interação com o consumidor em que este possa cocriar experiências únicas e desenvolver sua própria base de valor. Na perspectiva da experiência de marca, portanto, o consumidor deixa de ser apenas passivo às ações mercadológicas da empresa.

\subsubsection{Uma escala para brand experience}

Nos últimos anos, os estudos sobre brand experience têm atraído muita atenção de pesquisadores e profissionais da área de marketing. Walter, Cleff e Chu (2013) destacam que, a partir do entendimento de que os consumidores buscam não apenas benefícios funcionais de uma marca, mas experiências emocionais, a recente teoria de brand experience precisa proporcionar respostas sobre quanto medir brand experience e de que forma tais experiências afetam o comportamento do consumidor. O direcionamento de alguns desses estudos consiste tanto em buscar meios para entender e criar experiências que gerem valor agregado, quanto em propostas de medidas para avaliar resultados de experiências e definir estratégias competitivas de negócios (BRAKUS; SCHMITT; ZARANTONELLO, 2009; LEE; KANG, 2012; SHAMIM; BUTT, 2013; MORGAN-THOMAS; VELOUTSOU, 2013).

Entre os trabalhos que tratam de medidas, destaca-se um detalhado estudo feito por Brakus, Schmitt e Zarantonello (2009), os quais definiram uma escala para medir brand experience. Neste estudo, os autores examinaram empiricamente em que momento ocorrem as experiências, como elas podem afetar os julgamentos e atitudes, além de analisarem outros aspectos do comportamento do consumidor. As dimensões de brand experience foram definidas por meio de uma ampla e multidisciplinar revisão bibliográfica, abrangendo estudos de psicologia, ciência cognitiva, estudos aplica- 
dos sobre marketing experimental e gestão. Foi utilizada a técnica de análise fatorial confirmatória que indicou o melhor modelo para o construto brand experience, que consistiu em quatro fatores (sensorial, afetivo, comportamental e intelectual), como mostra o Esquema 1.

Esquema 1 - Modelo de quatro fatores para brand experience

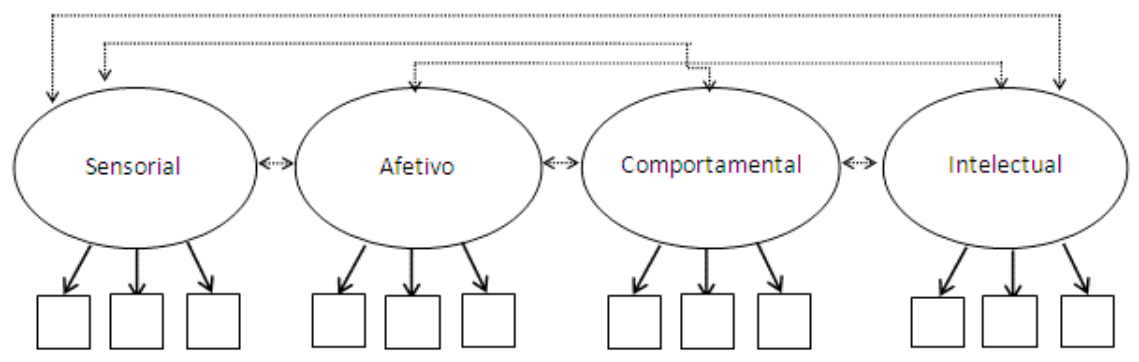

Fonte: adaptado de Brakus, Schmitt e Zarantonello (2009).

A escala proposta por Brakus, Schmitt e Zarantonello (2009) foi submetida a testes de validade e confiabilidade, além de análise discriminante em relação a outras escalas de marcas existentes na literatura. Após cinco fases do estudo, o modelo final consistiu em 12 itens distribuídos entre os fatores, conforme pode ser observado no Quadro 1.

Quadro 1 - Escala de Brand Experience

\begin{tabular}{|l|l|}
\hline \multicolumn{1}{|c|}{ Dimensão } & \multicolumn{1}{c|}{ Item } \\
\hline \multirow{5}{*}{ Sensorial } & Essa marca causa uma forte impressão no meu sentido visual e/ou em outros sentidos. \\
\cline { 2 - 3 } & Considero essa marca interessante de uma forma sensorial. \\
\cline { 2 - 3 } & Essa marca não apela aos meus sentidos. \\
\hline \multirow{5}{*}{$\begin{array}{l}\text { Comporta- } \\
\text { mental }\end{array}$} & Essa marca provoca emoções e sentimentos. \\
\cline { 2 - 3 } & Eu não tenho fortes emoções por essa marca. \\
\cline { 2 - 3 } Intelectual & Essa é uma marca que mexe com minhas emoções. \\
\cline { 2 - 3 } & Essa marca desperta em mim comportamentos e experiências sociais. \\
\cline { 2 - 3 } & Essa marca não é orientada para a ação. \\
\cline { 2 - 3 } & Eu me envolvo em pensamentos quando me deparo com essa marca. \\
\cline { 2 - 3 } & Essa marca não me faz pensar em nada. \\
\cline { 2 - 3 } & Essa marca estimula minha curiosidade e a resolução de problemas. \\
\hline
\end{tabular}

Fonte: adaptado de Brakus, Schmitt e Zarantonello (2009). 
A dimensão sensorial abrange os estímulos que fazem apelo aos sentidos do consumidor, ou seja, visão, olfato, tato, paladar e audição. Pontes (2012) exemplifica algumas das inúmeras situações nas quais as organizações podem desenvolver esses estímulos: nos produtos e suas embalagens, nos uniformes dos funcionários, nos ambientes e arquiteturas de estabelecimentos, em peças publicitárias, no sabor da comida, entre outras.

A dimensão afetiva envolve elementos que interferem no humor, nos sentimentos e nas emoções do indivíduo (SEMPREBOM, 2011). Para Pontes (2012), há variação na intensidade das experiências emocionais, conforme o estado de espírito do consumidor e a situação de consumo. O autor destaca que, ao entrar em uma experiência, o temperamento e a predisposição emocional de um indivíduo afetam a maneira e a intensidade dos seus sentimentos. Nesse caso, outros exemplos incluem sentimentos como divertimento, alegria, nostalgia ou frustração.

A dimensão comportamental está relacionada aos valores e crenças dos indivíduos, conforme o estilo de vida e comportamento. Schmitt (2002) menciona diversas categorias ligadas à ação: aspectos físicos, ações motoras, sinais corporais, influência do ambiente sobre o desejo físico, comportamento não verbal, estilo de vida, mudanças comportamentais, autopercepção e indução de ações não pensadas. Essas categorias estão divididas em dois grandes grupos: ações ligadas ao corpo e ações ligadas ao estilo de vida.

A dimensão intelectual está relacionada às experiências que estimulam o cliente a pensar, despertando sua criatividade e curiosidade, além de estimular o debate e gerar controvérsia (SABIOTE; BALLESTER, 2011). Essa é uma dimensão que envolve experiências que afetam mentalmente o consumidor. Pontes (2012) exemplifica diversas situações nas quais é possível perceber esse tipo de experiência. Para o autor, uma peça publicitária pode despertar diversas indagações no consumidor, uma vez que ele pode pensar se aquele produto possui alguma finalidade para ele, se realmente as informações que constam naquele folheto são verídicas ou se são apenas truques para induzi-lo à compra.

A escala de brand experience proposta por Brakus, Schmitt e Zarantonello (2009) forneceu duas significativas contribuições para estudiosos e profissionais da área de marketing. Por um lado, provou que brand experience afeta positivamente a lealdade e a satisfação do consumidor. Por outro lado, forneceu uma escala de brand experience, validada empiricamente, baseada nas dimensões sensorial, afetiva, comportamental e intelectual. 
A escala proposta por Brakus, Schmitt e Zarantonello (2009) representou um importante avanço na literatura de marketing, compondo trabalhos de pesquisadores em diferentes países. Na pesquisa de Walter, Cleff e Chu (2013), a escala foi aplicada para avaliar o impacto de brand experience na satisfação e na lealdade de consumidores da Alemanha e do Canadá com a marca BMW. Lee e Kang (2012), por sua vez, utilizaram apenas as dimensões afetiva e comportamental da escala de brand experience para investigar como essas dimensões afetam a qualidade do relacionamento com a marca entre consumidores na Coreia do Sul. Nysveen, Pedersen e Skard (2013), investigando o setor de serviços, validaram as quatro dimensões propostas pelos autores, mas também mostraram que para as marcas de serviços, uma quinta dimensão se mostrou relevante: a relacional. Para Schmitt, Brakus e Zarantonello (2015), a dimensão relacional, que defende a ideia de interações, pode tornar-se cada vez mais importante no futuro como as mídias e redes sociais, embora não tenham encontrado suporte empírico na pesquisa.

Apesar de se destacar a importância da escala de brand experience discutida no presente estudo, ressalta-se que há um forte predomínio de marcas norte-americanas e, portanto, é válido aplicá-la em outros contextos. Nessa perspectiva, Sabiote e Ballester (2011) partiram da escala original proposta por Brakus, Schmitt e Zarantonello (2009) e avaliaram brand experience utilizando marcas espanholas; Sahin, Zehir e Kitapçi (2011) realizaram um estudo entre consumidores de automóveis na Turquia, utilizando essa mesma escala para avaliar qual a relação entre os construtos confiança, lealdade e satisfação da marca, considerando e explorando os efeitos de brand experience sobre esses construtos.

No Brasil, ainda são raros os estudos empíricos que abordam o conceito multidimensional de brand experience. Um desses trabalhos foi apresentado por Semprebom (2011) e teve como objetivo validar a escala proposta por Brakus, Schmitt e Zarantonello (2009). Os resultados encontrados no estudo foram divergentes do modelo original. Diante disso, no presente trabalho se propõe contribuir com a ampliação desse campo de estudo, considerando aspectos relacionados ao contexto brasileiro.

\section{METODOLOGIA}

Com o propósito de testar a escala de brand experience, proposta por Brakus, Schmitt e Zarantonello (2009), em um contexto brasileiro, bem como comparar os resultados aos de outros estudos semelhantes já publicados, foi realizada uma pesquisa quantitativa e descritiva (GIL, 2002). O corte temporal proposto ao trabalho foi 
um estudo transversal que levanta informações de qualquer amostra dos partícipes somente uma vez (HAIR JUNIOR et al., 2009; MALHOTRA, 2001).

O modelo utilizado para a mensuração da experiência da marca foi adaptado de Brakus, Schmitt e Zarantonello (2009). Os autores desenvolveram esse modelo a partir de um estudo realizado em cinco etapas. Um dos resultados do trabalho dos autores foi um questionário composto de 12 itens, alocados em quatro dimensões preditoras de brand experience: sensorial, afetiva, comportamental e intelectual.

O instrumento de coleta de dados, portanto, foi um questionário estruturado, com aplicação presencial intermediada por computador, sendo o questionário desenvolvido e aplicado com o uso do software Qualtrics. As questões utilizadas no presente trabalho, portanto, são aquelas extraídas do trabalho de Brakus, Schmitt e Zarantonello (2009), que foram traduzidas utilizando-se back translation, técnica que garante uma tradução precisa e confiável do instrumento. A escala utilizada para os itens foi a mesma utilizada pelos autores do trabalho original: escala Likert de 7 pontos, variando de "discordo totalmente" a "concordo totalmente".

As marcas utilizadas no estudo foram uma adaptação das marcas do trabalho original de Brakus, Schmitt e Zarantonello (2009). Na terceira etapa do estudo dos autores, eles chegaram a 30 marcas experienciais, validadas por experts. Para aplicar a pesquisa no contexto brasileiro, as marcas internacionais de pouco apelo no Brasil foram substituídas. As marcas oriundas do estudo original, consideradas marcas experienciais também no contexto brasileiro foram Samsung e Nokia. Os autores testaram a marca iPod, que na presente pesquisa foi substituída pela marca Apple. Foram selecionadas 15 marcas experienciais adicionais com maior impacto no contexto brasileiro, correspondentes às marcas do estudo de Brakus, Schmitt e Zarantonello (2009): Visa, MacDonald's, Gilette, Coca-Cola, Fiat, Havaianas, Samsung, Apple, Nokia, Colgate, O Boticário, Nestlé, Sony, Google e Lacoste. Essas marcas foram submetidas a uma validação de conteúdo, baseada no julgamento de experts, para então serem aleatoriamente distribuídas em seis grupos de três marcas cada.

A população estudada no presente trabalho engloba estudantes universitários dos Cursos de Administração, Ciências Contábeis e Marketing de uma universidade situada em Mossoró, RN. A amostra não probabilística, por conveniência, foi composta por 254 respondentes. Cada respondente sorteava um link para um dos seis questionários disponíveis e analisava três marcas. Desse processo, foram obtidas 762 observações.

Os dados coletados foram utilizados para testar a validade convergente e discriminante das variáveis latentes preditoras do modelo, assim como a avaliação da confiabilidade composta desses construtos, por meio da Análise Fatorial Confirma- 
tória, a partir do software AMOS®. Uma vez que os construtos foram validados, foi realizado o teste de Análise de Variância (Anova), que objetiva determinar a probabilidade de que a diferença entre as médias dos grupos ocorra apenas em razão de erro amostral (HAIR JUNIOR et al., 2009). Assim, a partir do software SPSS ${ }^{2}$, procedeu-se com o teste, que possibilitou identificar a existência de diferença das dimensões de brand experience para cada marca analisada: a hipótese nula testada pela Anova foi se as médias das marcas analisadas, em cada dimensão de brand experience, são iguais.

A Anova aponta se há diferença de média de pelo menos um grupo em relação aos demais. Assim, para que se identifiquem quais médias são estatisticamente diferentes entre si, é necessário realizar um teste post hoc. Nesta pesquisa, uma vez encontrada a existência de diferenças entre os grupos, o teste HSD de Tukey foi utilizado para efetuar uma comparação post hoc da análise de variância (MAROCO, 2007), a fim de determinar quais grupos da amostra apresentam diferenças entre si. Assim, foram identificadas para cada marca as dimensões que mais aumentavam a brand experience.

\section{RESULTADOS E DISCUSSÕES}

A amostra da pesquisa foi composta, em sua maioria, por respondentes do sexo feminino (58,8\%). A faixa etária variou entre 16 e 48 anos; 48,8\% dos pesquisados estavam na faixa entre 20 e 25 anos.

Após a caracterização da amostra, foi realizada uma análise exploratória dos dados e foram detectados três outliers, que foram retirados da amostra, ficando esta composta por 759 observações. Não foram observados missing values na amostra. Os construtos do modelo são unidimensionais, sendo cada um deles formado por dois indicadores, uma vez que os itens de escala reversa foram retirados do modelo, tal como o modelo de Semprebom (2011). A normalidade multivariada pode ser observada por meio do exame da distribuição univariada: presença de outliers e parâmetros de assimetria e curtose. Kline (2005) indica que valores entre -2 e 2 são aceitáveis e indicam aproximada distribuição normal. Embora estudos das ciências sociais e comportamentais com frequência falhem em assumir a normalidade dos dados por meio do teste de Kolmogorov-Smirnov e/ou Shapiro-Wilks (MCDONALD; HO, 2002; YUAN; BENTLER, 2000), autores afirmam que os testes de estimação utilizados nos softwares de modelagem de equações estruturais são bastante robustos contra violações de normalidade (ANDERSON; GERBING, 1988; BROWNE; SHAPIRO, 1988; YUAN; BENTLER, 2000). Os valores absolutos de assimetria e curtose dos itens 
desta pesquisa ficaram entre -1 e +1 , indicando que os testes paramétricos podem ser utilizados (KLINE, 2005; MAROCO, 2010). A redução dos itens para quatro fatores foi corroborada pelo teste KMO, que apresentou resultado $0,813(\mathrm{p}<0,001)$.

Os valores médios obtidos e suas respectivas medidas de dispersão dos construtos apontam para uma percepção positiva em torno das dimensões sensorial, afetiva, comportamental e intelectual das marcas analisadas. As correlações bivariadas dos indicadores, para todas as variáveis latentes, foram positivas e significantes. Os Alfas de Cronbach encontrados para os construtos foram de 0,650 para a dimensão sensorial, 0,688 para a dimensão afetiva, 0,747 para a dimensão comportamental e 0,660 para a dimensão intelectual. Um dos fatores que justificam os baixos valores do alfa de Cronbach é o pequeno número de itens dos construtos. No entanto, estes podem ser considerados confiáveis, uma vez que em diversas áreas de conhecimento, principalmente nas ciências sociais, o Alfa de Cronbach de 0,6 é considerado aceitável (MALHOTRA, 2001).

Para verificar as variáveis dos construtos que compõem o modelo proposto, adotou-se a Análise Fatorial Confirmatória (CFA). O processo utilizado foi o método de estimação de máxima verossimilhança. O modelo testado, com quatro dimensões, com uma amostra de 759 observações, apresentou uma boa qualidade de ajustamento, conforme se observa na Tabela 1.

Tabela 1 - Medidas de ajuste da Análise Fatorial Confirmatória

\begin{tabular}{cccccrr}
\hline $\mathbf{x} 2 / \mathbf{g l}$ & CFI & IFI & NFI & RFI & \multicolumn{1}{c}{ RMSEA } & \multicolumn{1}{c}{ RMSR } \\
\hline $3,027^{* * *}$ & 0,983 & 0,983 & 0,975 & 0,951 & 0,05 & 0,0230 \\
\hline Fonte: os autores. & & & & & &
\end{tabular}

O modelo apresentou um $\chi^{2}$ de 42,384, com 14 graus de liberdade; assim, a relação $\chi^{2} / g l$ indica que o modelo está adequado (HAIR JUNIOR et al., 2009; MAROCO, 2010). As medidas de ajuste incremental apresentaram valores superiores a 0,9, conforme recomenda a literatura e, por fim, o RMSEA, representando o índice de discrepância e a análise dos resíduos estandardizados (RMSR), apresentou valores abaixo de 0,05, corroborando o ajuste do modelo.

As estimativas dos parâmetros dos caminhos dos construtos em relação às variáveis observadas são apresentadas na Tabela 2. Os resultados indicam significância estatística, já que são observadas razões críticas superiores a 1,96. 
Tabela 2 - Estimativas dos pesos de regressão, erro padrão e razão crítica do modelo

\begin{tabular}{|c|c|c|c|c|c|c|}
\hline \multicolumn{3}{|c|}{ Pesos de regressão } & \multirow{2}{*}{$\begin{array}{r}\text { Estimativa } \\
1,000\end{array}$} & \multirow[t]{2}{*}{ Erro padrão } & \multirow[t]{2}{*}{ Razão crítica } & \multirow[t]{2}{*}{$\mathbf{P}$} \\
\hline Q1_2 & $<---$ & Sensorial & & & & \\
\hline Q1_1 & $<---$ & Sensorial & 1,406 & ,136 & 10,335 & $* * *$ \\
\hline Q1_6 & $<---$ & Afetiva & 1,000 & & & \\
\hline Q1_4 & $<---$ & Afetiva & 1,012 & ,068 & 14,788 & $* * *$ \\
\hline Q1_8 & $<---$ & Comportamental & 1,000 & & & \\
\hline Q1_7 & $<---$ & Comportamental & 1,183 & 079 & 14,977 & $* * *$ \\
\hline Q1_12 & $<---$ & Intelectual & 1,000 & & & \\
\hline Q1_10 & $<---$ & Intelectual & 1,341 & 104 & 12,876 & $* * *$ \\
\hline
\end{tabular}

Fonte: os autores.

A Tabela 3, por sua vez, apresenta as estimativas das covariâncias entre os construtos. Observa-se, por meio da análise da razão crítica, que essas covariâncias apresentam significância estatística. Resultado semelhante foi destacado por Ozyer (2016), uma vez que todas as covariâncias entre as variáveis de experiência com marca foram significativas no seu estudo. Isso indica que as experiências sensoriais, afetivas, comportamentais e intelectuais ocorrem simultaneamente e afetam umas às outras. Por outro lado, autores como Semprebom (2011) e Walter, Cleff e Chu (2013) não obtiveram as mesmas categorias de análise em suas pesquisas: ambas as pesquisas não confirmaram a dimensão comportamental como fator determinante da experiência de marca, enquanto os resultados de Sabiote e Ballester (2011) apontam para a fusão das dimensões afetiva e comportamental.

Tabela 3 - Estimativas dos pesos de regressão, erro padrão e razão crítica do modelo

\begin{tabular}{lllllll}
\hline & & & Estimativa & Erro padrão & Razão crítica & P \\
\hline Sensorial & $<-->$ & Afetiva &, 669 &, 086 & 7,777 & $* * *$ \\
Sensorial & $<-->$ & Comportamental &, 550 &, 081 & 6,819 & $* * *$ \\
Intelectual & $<-->$ & Sensorial &, 676 &, 089 & 7,618 & $* * *$ \\
Afetiva & $<-->$ & Comportamental & 1,220 &, 121 & 10,050 & $* * *$ \\
Intelectual & $<-->$ & Afetiva & 1,126 &, 121 & 9,335 & $* * *$ \\
Intelectual & $<-->$ & Comportamental & 1,037 &, 119 & 8,700 & $* * *$ \\
\hline
\end{tabular}

Fonte: os autores.

Para a análise da validade e confiabilidade do modelo, verificou-se primeiramente o grau de ajustamento dos indicadores às dimensões propostas por meio de um indicador de Confiabilidade Composta, que permite atestar a consistência das medidas do modelo. Para testar a validade convergente, utilizou-se a Variância Média Extraída, que reflete a quantia geral de variância nos indicadores explicada pelo construto latente. Os resultados estão demonstrados na Tabela 4. 
Tabela 4 - Resultados da análise fatorial confirmatória, confiabilidade composta e variância média extraída dos construtos do modelo proposto

\begin{tabular}{lllll}
\hline Construtos Indicadores & Sensorial & Afetiva & Comportamental & Intelectual \\
\hline Q2 - Sensorial & 0,685 & & & \\
Q1 - Sensorial & 0,775 & & & \\
Q6 - Afetiva & & 0,726 & & \\
Q4 - Afetiva & & 0,772 & & \\
Q8 - Comportamental & & 0,709 & \\
Q7 - Comportamental & & 0,841 & 0,637 \\
Q12 - Intelectual & & & 0,848 \\
Q10 - Intelectual & & & & 0,72 \\
Confiabilidade Composta & 0,70 & 0,72 & 0,75 & 0,56 \\
Variância Média Extraída & 0,53 & 0,56 & 0,60 & \\
Fonte: os autores. & & & &
\end{tabular}

A confiabilidade composta dos fatores foi considerada satisfatória, pois tais valores devem ser superiores a 0,7. Os valores obtidos de variância média extraída, que representam a validade convergente do fator, foram satisfatórios, apresentando resultados superiores a 0,5 .

A validade discriminante indica que os construtos do modelo operacionalizam fatores diferentes (MAROCO, 2010). Para testar essa validade, foram verificadas as correlações entre as variáveis latentes, duas a duas. Os resultados estão expressos na Tabela 5.

Tabela 5 - Matriz de correlações e seus quadrados das variáveis latentes do modelo

\begin{tabular}{lrrrr}
\hline & \multicolumn{1}{c}{ Sensorial } & Afetiva & Comportamental & Intelectual \\
\hline Sensorial & 1 & & & \\
Afetiva & $0,544(0,295)$ & 1 & & \\
Comportamental & $0,441(0,187)$ & $0,686(0,471)$ & & 1 \\
Intelectual & $0,601(0,352)$ & $0,710(0,507)$ & $0,635(0,398)$ & 1 \\
Fonte: os autores. & & & &
\end{tabular}

Para Maroco (2010), a validade discriminante pode ser avaliada por meio da comparação entre o quadrado da correlação entre os fatores e a AVE de cada construto: caso a AVE dos fatores seja maior que o quadrado da correlação entre eles, pode-se afirmar que não há sobreposição de construtos. Assim, o quadrado da correlação entre as dimensões sensorial e afetiva é 0,295, que é inferior às variâncias médias extraídas dos fatores sensorial $(0,53)$ e afetivo $(0,56)$. Os demais construtos do modelo foram testados, de forma que se pode afirmar que o modelo não apresenta sobreposição de fatores. 
O modelo resultante do presente estudo, composto por quatro fatores, foi coerente ao estudo original e aos achados de Ozyer (2016). Embora tenham utilizado outra escala, o modelo de experiência de marca testado por Ding e Tseng (2015) consiste nas experiências de perceber (dimensão sensorial), sentir (dimensão afetiva), pensar (dimensão cognitiva), agir (dimensão comportamental), além da experiência de se relacionar (dimensão social), que não está presente no estudo de Brakus, Schmitt e Zarantonello (2009). Em relação aos trabalhos anteriores, o modelo do presente estudo apresentou melhores índices de ajuste em todos os quesitos em relação ao trabalho de tais autores e em três dos quatro índices testados em relação ao modelo de Semprebom (2011).

Após a análise do modelo geral, as marcas foram elencadas em termos de média geral e média por construto. Para determinar se a diferença de médias de brand experience e suas dimensões é estatisticamente significante entre as marcas da pesquisa, realizou-se o teste de Análise de Variância (Anova). Os referidos dados estão dispostos na Tabela 6.

Tabela 6 - Médias das marcas avaliadas, por construto, e análise de variância

\begin{tabular}{|c|c|c|c|c|c|c|}
\hline & & $\begin{array}{c}\text { Brand ex- } \\
\text { perience }\end{array}$ & Sensorial & Afetiva & $\begin{array}{l}\text { Compor- } \\
\text { tamental }\end{array}$ & $\begin{array}{c}\text { Intelec- } \\
\text { tual }\end{array}$ \\
\hline \multicolumn{2}{|c|}{ Média (DP) do construto } & 4,20 & 5,14 & 3,98 & 3,62 & 4,05 \\
\hline \multirow{18}{*}{$\begin{array}{l}\text { Médias das } \\
\text { marcas ava- } \\
\text { liadas }\end{array}$} & O Boticário & 4,67 & 5,21 & 5,16 & 4,34 & 3,97 \\
\hline & Ray-Ban & 4,65 & 6,03 & 4,36 & 4,02 & 4,20 \\
\hline & Google & 4,51 & 5,30 & 3,53 & 3,42 & 5,77 \\
\hline & Nestlé & 4,48 & 5,22 & 4,60 & 4,12 & 3,97 \\
\hline & Nokia & 4,45 & 5,40 & 3,84 & 3,88 & 4,70 \\
\hline & Kibon & 4,41 & 5,56 & 4,63 & 3,62 & 3,82 \\
\hline & Apple & 4,39 & 5,48 & 4,22 & 3,06 & 4,78 \\
\hline & Lacoste & 4,38 & 5,30 & 4,06 & 3,98 & 4,17 \\
\hline & Microsoft & 4,23 & 5,51 & 3,78 & 3,12 & 4,49 \\
\hline & Sony & 4,19 & 5,24 & 3,93 & 3,45 & 4,16 \\
\hline & Fiat & 4,15 & 4,95 & 3,72 & 3,78 & 4,14 \\
\hline & Samsung & 4,11 & 5,16 & 3,95 & 3,33 & 4,00 \\
\hline & Gillette & 4,05 & 4,65 & 3,46 & 4,50 & 3,58 \\
\hline & Visa & 4,00 & 4,31 & 3,72 & 3,59 & 4,40 \\
\hline & Colgate & 3,93 & 5,05 & 3,64 & 3,67 & 3,38 \\
\hline & Coca-Cola & 3,72 & 4,83 & 3,93 & 2,96 & 3,17 \\
\hline & Havaianas & 3,65 & 4,82 & 3,43 & 3,41 & 2,94 \\
\hline & MacDonald's & 3,65 & 4,58 & 3,78 & 2,95 & 3,28 \\
\hline Teste F & & 3,213 & 3,661 & 3,193 & 3,523 & 7,864 \\
\hline Sig. & & ,000 & ,000 & ,000 & ,000 & ,000 \\
\hline
\end{tabular}


Os itens que apresentaram maiores médias foram aqueles referentes à dimensão sensorial, enquanto a dimensão que apresentou a pior média foi a dimensão comportamental. Observa-se também menor dispersão das respostas em relação à dimensão sensorial, o que indica a relevância dos sentidos na experiência de consumo para a maioria dos consumidores pesquisados.

Partindo do princípio de que todas as marcas foram consideradas experimentais, a que apresentou maior experiência de marca foi O Boticário, enquanto McDonald's apresentou a pior experiência de marca entre as marcas testadas. É válido ressaltar que a O Boticário foi apontada como a marca com maior experiência de consumo em outros trabalhos realizados, como a pesquisa de Semprebom (2011). O autor justifica o bom desempenho dessa marca a partir dos altos índices obtidos nas dimensões sensorial e afetiva, fato que também se observa no presente trabalho.

As seis marcas que apresentaram os piores resultados de brand experience são relacionadas ao consumo corrente: MacDonald's, Coca-Cola, Havaianas, Colgate, Visa e Gillette. Outros itens de consumo corrente (O Boticário, Nestlé e Kibon) ficaram entre as seis melhores marcas experienciais. $\mathrm{O}$ que diferencia o comportamento destas em detrimento das marcas de pior resultado é em parte atribuído ao valor obtido na dimensão sensorial, que teve grande impacto na brand experience, e também ao valor obtido na dimensão afetiva: O Boticário, Nestlé e Kibon são marcas facilmente ligadas ao afeto e às emoções, sendo estas duas últimas marcas que evocam o imaginário da infância.

Os resultados da Anova demonstram que, em um nível de significância de até 1\%, deve-se rejeitar a hipótese nula de que as médias não diferem estatisticamente entre si. Quanto à brand experience, o teste HSD de Tukey identificou três conjuntos de marcas, com médias estatisticamente diferentes entre si, que foram categorizados em grupos, como apregoam Brakus, Schmitt e Zarantonello (2009): as marcas com maiores médias foram categorizadas como únicas, as marcas com valores intermediários foram categorizadas como altamente diferenciadas e as marcas com pior resultado foram classificadas como moderadamente diferenciadas. O Quadro 2 mostra as marcas pesquisadas, divididas por grupos em relação à brand experience. 
Quadro 2 - Grupos de marcas categorizados pelas médias obtidas na brand experience

\begin{tabular}{|l|l|l|l|l|}
\hline \multicolumn{1}{|c|}{$\begin{array}{c}\text { Moderadamente } \\
\text { diferenciadas }\end{array}$} & \multicolumn{3}{c|}{$\begin{array}{c}\text { Altamente } \\
\text { diferenciadas }\end{array}$} & \multicolumn{1}{c|}{ Únicas } \\
\hline McDonald's & Visa & Sony & Kibon & Nestlé \\
\hline Coca-Cola & Gillette & Microsoft & Nokia & Google \\
\hline Havaianas & Fiat & Lacoste & & Ray-Ban \\
\hline Colgate & Samsung & Apple & & O Boticário \\
\hline
\end{tabular}

Fonte: os autores.

De acordo com os dados demonstrados no Quadro 2, percebeu-se que as marcas que se enquadraram em moderadamente diferenciadas são de pouco valor agregado e/ou consumo imediato. O fator simbólico, que transforma marcas em elementos de integração social (BOURDIEU, 1989), não se destaca como fator que diferencia as marcas únicas: a única marca com alto poder simbólico, dentro da categoria marcas únicas, é a Ray-Ban. As marcas mais aparentes, que podem demonstrar determinado status para o grupo, estão, em sua maioria, categorizadas nas marcas altamente diferenciadas.

Em relação às marcas ligadas a produtos físicos tecnológicos (Nokia, Samsung, Sony, Apple), os resultados obtidos no presente estudo divergem dos achados de Brakus, Schmitt e Zarantonello (2009). Neste, à exceção do iPod (Apple), tais marcas não foram caracterizadas como experiências diferenciadas, enquanto no contexto brasileiro, elas estão no grupo de experiências altamente diferenciadas, o que indica que têm um valor diferenciado para o consumidor brasileiro.

Além de serem categorizadas quanto à brand experience, observou-se como as marcas se posicionaram em cada dimensão, dentro das categorias moderadamente diferenciadas, altamente diferenciadas e marcas únicas. A separação das marcas nos grupos foi realizada pelo teste HSD de Tukey. O resultado da classificação está disposto no Quadro 3. 
Quadro 3-Grupos de marcas categorizados pelas dimensões da pesquisa

\begin{tabular}{|c|c|c|c|}
\hline Dimensão & Moderadamente diferenciada & Altamente diferenciada & Única \\
\hline \multirow{12}{*}{ Sensorial } & \multirow{12}{*}{ Visa } & \multirow{3}{*}{ McDonald's } & Colgate \\
\hline & & & O Boticário \\
\hline & & & Nestlé \\
\hline & & \multirow{2}{*}{ Gillette } & Sony \\
\hline & & & Samsung \\
\hline & & \multirow{2}{*}{ Coca-Cola } & Google \\
\hline & & & Lacoste \\
\hline & & \multirow{2}{*}{ Fiat } & Nokia \\
\hline & & & Apple \\
\hline & & \multirow{3}{*}{ Havaianas } & Microsoft \\
\hline & & & Kibon \\
\hline & & & Ray-Ban \\
\hline \multirow{11}{*}{ Afetiva } & & Havaianas & Samsung \\
\hline & & Gillette & Lacoste \\
\hline & & Google & Apple \\
\hline & & Colgate & \multirow{2}{*}{ Ray-Ban } \\
\hline & & Fiat & \\
\hline & & Visa & \multirow{2}{*}{ Nestlé } \\
\hline & & McDonald's & \\
\hline & & Microsoft & \multirow{2}{*}{ Kibon } \\
\hline & & Nokia & \\
\hline & & Coca-Cola & \multirow{2}{*}{ O Boticário } \\
\hline & & Sony & \\
\hline Comportamental & $\begin{array}{l}\text { McDonald's } \\
\text { Coca-Cola }\end{array}$ & $\begin{array}{l}\text { Apple } \\
\text { Microsoft } \\
\text { Samsung } \\
\text { Google } \\
\text { Sony } \\
\text { Havaianas } \\
\text { Visa } \\
\text { Kibon } \\
\text { Colgate } \\
\text { Fiat } \\
\text { Nokia } \\
\text { Lacoste } \\
\text { Ray-Ban } \\
\text { Nestlé }\end{array}$ & Gillette \\
\hline
\end{tabular}




\begin{tabular}{|c|c|c|c|}
\hline Dimensão & Moderadamente diferenciada & Altamente diferenciada & Única \\
\hline \multirow{9}{*}{ Intelectual } & \multirow{2}{*}{ Havaianas } & Gillette & \multirow{2}{*}{ Visa } \\
\hline & & Kibon & \\
\hline & \multirow{2}{*}{ Coca-Cola } & Nestlé & \multirow{2}{*}{ Microsoft } \\
\hline & & O Boticário & \\
\hline & \multirow{3}{*}{ McDonald's } & Samsung & \multirow{2}{*}{ Nokia } \\
\hline & & Fiat & \\
\hline & & Sony & \multirow{2}{*}{ Apple } \\
\hline & \multirow{2}{*}{ Colgate } & Lacoste & \\
\hline & & Ray-Ban & Google \\
\hline
\end{tabular}

Fonte: os autores.

De acordo com os dados demonstrados no Quadro 3, percebeu-se que a marca que apresentou menor apelo aos sentidos foi a marca Visa, enquanto a maioria delas foi reportada com forte apelo sensorial. Destaca-se, mais uma vez, que as médias das marcas foram elevadas e apresentaram baixa dispersão no que se refere à dimensão sensorial, o que explica esse alto desempenho.

Na dimensão afetiva, nenhuma das marcas foi identificada como moderadamente diferenciada; a maioria delas foi enquadrada como altamente diferenciada. Observa-se que Apple e Samsung, que dividem a liderança no segmento de smartphones e tablets no Brasil, figuram como as duas únicas marcas ligadas à tecnologia categorizadas como marcas únicas.

Para os consumidores testados, apenas O Boticário e Gillette se caracterizaram como marcas únicas, são as marcas que mais influenciam seu comportamento e proporcionam opções de estilo de vida. As marcas que têm menor influência no comportamento foram McDonald's e Coca-Cola, enquanto as demais foram identificadas como altamente diferenciadas.

Por fim, na categoria intelectual, as marcas ficaram mais distribuídas nas três categorias, visto que a maior parte delas foi enquadrada como altamente diferenciada. Percebe-se a predominância de marcas ligadas à tecnologia entre as marcas únicas, o que corrobora a ideia de envolvimento mental do consumidor na situação de consumo.

Brakus, Schmitt e Zarantonello (2009) destacaram que embora algumas marcas tenham um relacionamento positivo e duradouro com os consumidores, esse relacionamento não se traduz em experiências únicas. Esse fato pode ser observado, na presente pesquisa, com a Coca-Cola, que não apareceu como marca única em nenhuma das dimensões da brand experience. 


\section{CONSIDERAÇÕES FINAIS}

Nesta pesquisa procurou-se contribuir com a área de marketing por meio da validação da escala de brand experience no contexto brasileiro, escala que pode colaborar com os gestores na elaboração dos estímulos certos, no sentido de promover o relacionamento da marca com os clientes de forma positiva. A compreensão sobre brand experience pode conduzir para uma melhor tomada de decisão em relação à direção estratégica da empresa para o posicionamento da sua marca por meio da identificação das dimensões que tornam essa marca diferenciada ou única, e construindo uma relação de consumo de longo prazo. A escala testada foi proposta por Brakus, Schmitt e Zarantonello (2009) e compreende a experiência de marca em quatro dimensões: sensorial, afetiva, comportamental e intelectual. A pesquisa objetivou, também, comparar os resultados aos de outros estudos semelhantes já publicados.

A dimensão sensorial apresentou as maiores médias enquanto a dimensão comportamental apresentou as mais baixas. De forma geral, as duas dimensões que apresentaram maior impacto na experiência de consumo foram a sensorial e a afetiva. Um maior impacto da dimensão afetiva foi decorrente do fato de não haver nenhuma marca no grupo moderadamente diferenciada, já as demais dimensões apresentaram maior incidência de marcas no grupo altamente diferenciadas.

No que se refere à brand experience, percebeu-se que as marcas que apresentaram menor grau de diferenciação são aquelas de pouco valor agregado e/ou consumo imediato. As marcas que são conhecidas por conferir determinado grau de status ao consumidor (à exceção da Ray-Ban), como veículos, itens de vestuário e especialmente produtos eletrônicos, foram categorizadas como altamente diferenciadas, e isso é uma característica do mercado nacional, não tendo surgido de forma tão contundente nas pesquisas internacionais. As marcas únicas foram aquelas com forte apelo sensorial e/ou afetivo, como O Boticário, Nestlé, Ray-Ban e Google.

Estudos relacionados à brand experience não são tradicionais na literatura acadêmica; no entanto, eles representam tema relevante na área de marketing, uma vez que a experiência implica diretamente o envolvimento do consumidor, em diferentes níveis, com uma marca. Nesse sentido, neste estudo apresentou-se uma contribuição ao esforço de validar uma escala de mensuração de brand experience o contexto brasileiro. Vale ressaltar que trabalhos de mensuração por escala são instrumentos úteis, mas podem simplificar a análise de determinado fenômeno.

As principais contribuições do presente estudo à área de conhecimento relativa à experiência com a marca são: neste trabalho oferecem-se evidências empíricas 
de que as empresas devem gerenciar as experiências com marcas considerando que há ocasiões em que essas experiências são mais memoráveis do que o próprio ato do consumo; as duas dimensões que tiveram maior impacto na experiência de marca, sendo importantes diretrizes para o desenvolvimento de estratégias de construção e fortalecimento de experiências de marcas são sentidos e afetividade; em relação ao contexto nacional, os consumidores brasileiros demonstraram uma experiência de marca mais memorável com seus produtos tecnológicos em relação a consumidores norte-americanos, e a causa dessa diferença parece residir no fator simbólico, que transforma essas marcas em elos de integração social.

Recomenda-se que as empresas invistam mais na interação dos seus produtos com os sentidos dos consumidores, uma vez que essa dimensão apresentou forte impacto na experiência de consumo. Limitações do estudo podem estar associadas à amostra selecionada. Cabe considerar que os dados obtidos dizem respeito a estudantes de nível superior, o que pode causar algum viés. Nesse sentido, recomenda-se a ampliação desta pesquisa com grupos de entrevistados distintos. Sugerem-se, ainda, o desenvolvimento e o aprofundamento da escala de brand experience em segmentos específicos, como artigos de luxo e equipamentos eletrônicos, para mensurar o grau de influência das dimensões sobre a experiência de marca.

\section{REFERÊNCIAS}

AAKER, J. L. Dimensions of Brand Personality. Journal of Marketing Research, v. 34, i. 3, p. 347, ago. 1997.

ANDERSON, J. C.; GERBING, D. W. Structural equation modeling in practice: A review and recommended two-step approach. Psychological Bulletin, v. 103, p. 411-423, 1988.

BOURDIEU, P. O poder simbólico. Lisboa, Portugal: Difel, 1989.

BRAKUS, J. J.; SCHMITT, B. H.; ZARANTONELLO, L. Brand Experience: What Is It? How Is It Measured? Does It Affect Loyalty? Journal of Marketing, v. 73, i. 3, p. 52-68, maio 2009.

BREIVIK, E.; THORBJØRNSEN, H. Consumer brand relationships: an investigation of two alternative models. Journal of the Academy of Marketing Science, v. 36, i. 4, p. 443-472, ago. 2008. 
BROWNE, M. W.; SHAPIRO, A. Robustness of Normal Theory Methods in the Analysis of Linear Latent Variate Models. British Journal of Mathematical and Statistical Psychology, v. 41, p. 193-208, 1988.

DING, C. G.; TSENG, T. H. On the relationships among brand experience, hedonic emotions, and brand equity. European Journal of Marketing, v. 49, i. 7-8, p. 9941015, 2015.

FOURNIER, S. Consumers and their brands: developing relationship theory in consumer research. Journal of consumer research, v. 24, p. 343-373, Mar. 1998.

GENTILE, C.; SPILLER, N.; NOCI, G. How to Sustain the Customer Experience: European Management Journal, v. 25, i. 5, p. 395-410, Oct. 2007.

GIL, A. C. Como elaborar Projetos de Pesquisa. 4. ed. São Paulo: Atlas, 2002.

HAIR JUNIOR, J. F. et al. Análise multivariada de dados. Porto Alegre: Bookman, 2009.

HEDING, T.; KNUDTZEN, C.; BJERRE, M. Brand Management. [S.l.]: Routledge, 2009.

HOLBROOK, M. B.; HIRSCHMAN, E. C. The experiential aspects of consumption: consumer fantasies, feelings, and fun. Journal of consumer research, v. 9, i. 2, p. 132-140, 1982.

HOLBROOK, M. B. The Millennial Consumer in the Texts of Our Times: Experience and Entertainment. Journal of Macromarketing, v. 20, i. 2, p. 178-192, Dec. 2000.

IGLESIAS, O.; SINGH, J. J.; BATISTA-FOGUET, J. M. The role of brand experience and affective commitment in determining brand loyalty. Journal of Brand Management, v. 18, i. 8, p. 570-582, 2011.

KAPFERER, J.-N. The new strategic brand management: creating and sustaining brand equity long term. 4. ed. London: Kogan Page, 2008.

KELLER, K. Brand synthesis: The multidimensionality of brand knowledge. Journal of consumer research, v. 29, p. 595-600, Mar. 2003. 
KLINE, R. B. Principles and practice of structural equation modeling. New York: Guilford Press, 2005.

LEE, H. J.; KANG, M. S. The effect of brand experience on brand relationship quality. Academy of Marketing Studies Journal, v. 16, i. 1, p. 87-98, 2012.

MALHOTRA, N. Pesquisa de marketing: uma orientação aplicada. Porto Alegre: Bookman, 2001.

MAROCO, J. Análise de Equações Estruturais: fundamentos teóricos, software \& aplicações. Pêro Pinheiro: Reportnumber, 2010.

MAROCO, J. Análise estatística com utilização do SPSS. 3. ed. Lisboa, Portugal: Edições Sílabo, 2007.

MCDONALD, R. P.; HO, M. R. Principles and practice in reporting structural equation analyses. Psychological methods, v. 7, i. 1, p. 64-82, 2002.

MORGAN-THOMAS, A.; VELOUTSOU, C. Beyond technology acceptance: Brand relationships and online brand experience. Journal of Business Research, v. 66, i. 1, p. 21-27, Jan. 2013.

MUNIZ, A. M. J.; O’GUINN, T. C. Brand Community. Journal of Consumer Research, v. 27, p. 412-432, Mar. 2001.

MUNIZ, K. M.; MARCHETTI, R. Z. Brand personality dimensions in the Brazilian context. BAR. Brazilian Administration Review, v. 9, i. 2, p. 168-188, June 2012.

NYSVEEN, H.; PEDERSEN, P. E.; SKARD, S. Brand experiences in service organizations: Exploring the individual effects of brand experience dimensions. Journal of Brand Management, i. 20, p. 404-23, 2013.

OZYER, Y. Understanding the Impact of the Brand Experience on Brand Reputation by the Moderating Role of Technology Turbulence. International Journal of Marketing Studies, v. 8, i. 1, p. 161-169, 2016.

PINE, B.; GILMORE, J. Welcome to the experience economy. Harvard business review, 1998. 
PONTES, M. C. Marketing experiencial ou o uso da experiência no Marketing? Estudo de caso em hotéis 5 estrelas da cidade de São Paulo. 2012. Tese (Doutorado em Administração)-Universidade de São Paulo, São Paulo, 2012.

PRAHALAD, C. K.; RAMASWAMY, V. Co-creation experiences: The next practice in value creation. Journal of Interactive Marketing, v. 18, i. 3, p. 5-14, Jan. 2004.

QADER, I. K. A.; OMAR, A. B. The Evolution of Experiential Marketing: Effects of Brand Experience among the Millennial Generation. International Journal of Academic Research in Business and Social Sciences, v. 2, i. 1, p. 331-340, July 2012.

SABIOTE, E. F.; BALLESTER, M. E. D. Marcas de experiencia: marcando la diferencia. Estudios Gerenciales, v. 27, n. 121, p. 59-77, 2011.

SAHIN, A.; ZEHIR, C.; KITAPÇI, H. The Effects of Brand Experiences, Trust and Satisfaction on Building Brand Loyalty; An Empirical Research On Global Brands. Procedia - Social and Behavioral Sciences, v. 24, p. 1288-1301, Jan. 2011.

SCHMITT, B. H.; BRAKUS, J.; ZARANTONELLO, L. The current state and future of brand experience. Journal of Brand Management, v. 21, i. 9, p. 727-733, 2015.

SCHMITT, B. H. Marketing Experimental. São Paulo: Nobel, 2002.

SEMPREBOM, E. Experiência com Marca: Validação da Escala no Contexto Universitário. In: ENCONTRO DA ANPAD, 35., 2011, Rio de Janeiro. Anais... Rio de Janeiro, 2011.

SHAMIM, A.; BUTT, M. M. A critical model of brand experience consequences. Asia Pacific Journal of Marketing and Logistics, v. 25, i. 1, p. 102-117, 2013.

WALTER, N.; CLEFF, T.; CHU, G. Brand experience’s influence on customer satisfaction and loyalty: a mirage in marketing research? International Journal of Management Research and Business Strategy, v. 2, i. 1, 2013.

YUAN, K.-H.; BENTLER, P. M. Three Likelihood-Based Methods For Mean and Covariance Structure Analysis With Nonnormal Missing Data. Sociological Methodology, v. 30, i. 1, p. 165-200, 2000. 


\section{Como citar este artigo:}

\section{ABNT}

BRITO, Simone Gurgel de et al. Dimensões de brand experience no contexto brasileiro. RACE, Revista de Administração, Contabilidade e Economia, Joaçaba: Ed. Unoesc, v. 16, n. 1, p. 121-146, jan./abr. 2017. Disponível em: <http://editora. unoesc.edu.br/index.php/race>. Acesso em: dia/mês/ano.

APA

Brito, S. G. de, Oliveira, A. M. de, Nobre, L. H. N., Macêdo, A. F. P. de, \& Marchetti R. Z. (2017). Dimensões de brand experience no contexto brasileiro. RACE, Revista de Administração, Contabilidade e Economia, 16(1), 121-146. Recuperado em dia/ mês/ano, de http://editora.unoesc.edu.br/index.php/race 